\begin{tabular}{r|r|r|r|r|r|}
\hline Revista Duazary & ISSN: $1794-5992$ & Vol. 13 & No. 2 & $87-94$ & Julio - Diciembre de 2016 \\
\hline \multicolumn{6}{c|}{ DOI: http://dx.doi.org/10.21676/2389783X.1713 }
\end{tabular}

\title{
CONTEO DE PLAQUETAS EN SUJETOS SANOS TRATADOS CON ANTIAGREGANTES PLAQUETARIOS
}

\author{
PLATELET COUNT IN HEALTHY SUBJECTS TREATED WITH \\ ANTIPLATELET DRUGS
}

TÍTULO CORTO: CONTEO DE PLAQUETAS EN SUJETOS SANOS

\section{Maczy González-Rincón', Melvis Arteaga-Vizcaino² ${ }^{1}$ Ana Ruíz ${ }^{3}$, Olga Briceño ${ }^{4}$, Maribel Quintero ${ }^{5}$, Jesús Quintero ${ }^{6}$, José Ramón Urdaneta ${ }^{7}$}

Recibido en agosto 14 de 2015

Aceptado en noviembre 19 de 2015

\section{RESUMEN}

Se evaluó el recuento plaquetario en sangre periférica de sujetos sanos tratados con antiagregantes plaquetarios. Se analizaron 20 sujetos. Se distribuyeron en dos grupos: $\underline{\mathrm{A}}$ : 10 sujetos que recibieron Aspirina (100 mg) y $\underline{\mathrm{B}}$ : $10 \mathrm{con}$ Clopidogrel $(75 \mathrm{mg})$ por 7 días. Se les realizó recuento plaquetario en sangre periférica y en PRP. Encontrándose un conteo de plaquetas antes del tratamiento con antiagregantes en sangre periférica de 258,6 $\pm 54,46 \times 10^{9} 17$ días después de $254 \pm 41,86 \times 10^{9} 1$ (Aspirina), 285,4 \pm 70,92 y 196,5 $\pm 37,90 \times 10^{9} 1$ (Clopidogrel) respectivamente. En el PRP de los sujetos antes de recibir Aspirina fue 486,5 $\pm 129,54 \times 10^{9} 1$ y después 449,2 $\pm 85,51 \times 10^{9} \mathrm{l}$; antes de la ingestión de Clopidogrel fue 565,2 \pm 150,41 y 592,9 $\pm 203,46$ x $10^{9} 1$ después del tratamiento. Se encontraron diferencias significativas solo para el conteo plaquetario en el grupo del Clopidogrel $(\mathrm{p}<0.05)$. Se observó una disminución significativa del conteo plaquetario en sangre periférica posterior a la administración del Clopidogrel, posiblemente como consecuencia del mecanismo farmacológico del mismo. Son necesarios más estudios para evaluar un mayor número de individuos y medir mejor el efecto de los antiplaquetarios.

Palabras clave: Plasma rico en plaquetas; Aspirina; Clopidogrel

1. Doctora en Ciencias de la Salud, docente titular a dedicación exclusiva, Jefa del Departamento de Morfofisiopatologia, Escuela de Bioanálisis, Universidad del Zulia, LUZ, Venezuela. Correo: maczygonzalez@hotmail.com; maczy.gonzalez@gmail.com

2. Doctora en Ciencias Médicas, docente titular a dedicación exclusiva e investigadora del Instituto de Investigaciones Clínicas Dr. Américo Negrette, Facultad de Medicina, Universidad del Zulia, LUZ, Venezuela. Correo: melvisarteaga@gmail.com

3. Doctora en Ciencias de la Salud, docente titular a dedicación exclusiva de la Catedra de Hematología, Escuela de Bioanálisis, Universidad del Zulia, LUZ, Venezuela. Correo: ruizag2801@yahoo.es

4. Doctora en Bioquímica y Genética, docente titular a dedicación exclusiva de la Catedra de Hematología, Escuela de Bioanálisis, Universidad del Zulia, LUZ, Venezuela. Correo: olguibp@yahoo.es

5. Doctora en Ciencias de la Salud, docente titular a dedicación exclusiva, Jefa de la Catedra Practica Profesional de Hematología, Escuela de Bioanálisis, Universidad del Zulia, LUZ, Venezuela. Correo: maribelquintero610@hotmail.com

6. Doctor en Ciencias Médicas, docente titular a dedicación exclusiva e investigador en jefe de la Sección de Hematología del Instituto de Investigaciones Clínicas Dr. Américo Negrette, Facultad de Medicina, Universidad del Zulia, LUZ, Venezuela. Correo: jequin@gmail.com

7. Doctor en Ciencias Médicas, docente agregado a medio tiempo de la Catedra de Anatomía, Escuela de Bioanálisis, Universidad del Zulia, LUZ, Venezuela. Correo: doctorjrum@hotmail.com 


\section{ABSTRACT}

Platelet count in peripheral blood of healthy subjects with antiplatelet drugs. 20 subjects were analized. They were distributed in two groups: subject $\underline{\mathrm{A}}$ : 10 who received aspirin $(100 \mathrm{mg})$ and $\underline{\mathrm{B}}: 10$ with Clopidogrel $(75 \mathrm{mg})$ for 7 days. In all subjects studied platelet count in peripheral blood and PRP. It found a platelet count before treatment with antiplatelet agents in peripheral blood of $258,6 \pm 54,46 \times 10^{9} \mathrm{l}$ and 7 days after $254 \pm 41,86 \times 10^{9} \mathrm{l}$ (aspirin) and $285,4 \pm 70,196,5 \pm 37,90 \times 10^{9} \mathrm{l}$ (Clopidogrel) respectively. In the PRP of subjects before receiving aspirin was $486,5 \pm 129,54 \times 10^{9} \mathrm{l}$ and after 449,2 $\pm 85,51 \times 10^{9} \mathrm{l}$; prior to Clopidogrel ingestion was 565,2 $\pm 150,41$ and 592,9 $\pm 203,46 \times 10^{9} \mathrm{l}$ after treatment. Significant differences were found only for the platelet count in the Clopidogrel Group ( $\mathrm{p}<0.05$ ). A significant decrease in platelet count was observed in peripheral blood after administration of Clopidogrel, possibly as a result of its pharmacological mechanism. More studies are needed to assess a greater number of individuals and better measure the effect of antiplatelet agents.

Keywords: Platelet-Rich Plasma; Aspirin; Clopidogrel

\section{INTRODUCCIÓN}

$\mathrm{L}$ as plaquetas son fragmentos citoplasmáticos provenientes de los megacariocitos de la médula ósea, sin núcleo y por ende tampoco ADN genómico, sin embargo, contienen ARN mensajero derivado de sus predecesores inmediatos, que lleva a cabo la síntesis de las proteínas. Tienen forma discoide, con dimensiones de 2 a 4 por $0,5 \mu \mathrm{m}$, circulan en concentraciones de $150-450$ plaquetas $\times 10^{9} \mathrm{l}$; el $70 \%$ se mantiene en circulación mientras que el otro $30 \%$ permanece de manera transitoria, pero constante, en el bazo y en la circulación por un promedio de 10 a 11 días $^{1}$. Contienen gránulos secretorios como los $\alpha$, que determinan su valor funcional por las sustancias que se segregan una vez que las plaquetas han sido activadas. También poseen una alta concentración de factores de crecimiento (FCs), que ha llevado a considerar el uso de Plasma Rico en Plaquetas (PRP) como potenciador de los mecanismos de regeneración tisular, sin producir efectos negativos ${ }^{2-4}$.

El PRP es una fracción plasmática con altas concentraciones de plaquetas, de 2 a 5 veces su número en sangre periférica, cuyo valor promedio es alrededor de 200 plaquetas x $10^{3} / \mu l$. Una concentración de $1.000 \times 10^{3} /$ $\mu \mathrm{l}$ plaquetas, es ideal para asegurar un aporte óptimo de FCs y estimular la consolidación de huesos y tejidos blandos ${ }^{5}$. En ese sentido, se han realizado diversos estudios para determinar el recuento plaquetario tanto en sangre periférica como en PRP, para establecer el porcentaje de recuperación plaquetaria en cada producto; sin embargo, poco se ha estudiado de manera exhaustiva, sobre cómo se afecta este conteo en sujetos bajo tratamiento con antiagregantes plaquetarios ${ }^{6,7}$. Hoy día, la aterogénesis y trombogénesis arterial es tema de estudio de todas las especialidades relacionadas con el diagnóstico, tratamiento y prevención de las enfermedades aterotrombóticas ${ }^{8}$, y entre los medicamentos empleados están los antiagregantes plaquetarios.

Los conocimientos obtenidos acerca de la estructura, ultraestructura y características funcionales plaquetaria, han permitido una mejor comprensión de su fisiología, así como la acción de fármacos con función antiagregantes, tan antiguo como la Aspirina (Ácido Acetil Salicilico-AAS), y otras nuevas drogas como las Ticlopidina, Tienopiridinas, un derivado de esta última es el Clopidogrel. La AAS y el Clopidogrel, son los antiagregantes plaquetarios que han sido recomendados para reducir el riesgo de eventos vasooclusivos recurrentes en pacientes con enfermedad cardiovascular?.

La AAS inhibe la formación de tromboxano $\mathrm{A}_{2}$ en la plaqueta y de Prostaciclina en el endotelio vascular; y el Clopidogrel actúa inhibiendo los receptores plaquetarios del ADP9. La acción de estas drogas se mantiene durante toda la vida de la plaqueta (8-10 días) ${ }^{10}$; la AAS no ejerce un efecto antiplaquetario uniforme en todas 
las plaquetas pues su acción está sujeta a variables individuales (factores clínicos, celulares y genéticos) ${ }^{11}$. Aun cuando la tolerancia para ambas drogas es similar, las hemorragias gastrointestinales son menos frecuentes con el Clopidogrel ${ }^{8}$.

En pacientes tratados con Clopidogrel o aspirina, se ha reportado una variedad de efectos hematológicos en sangre periférica que incluyen trombocitopenia ${ }^{12,13}$. En estudios previos también se ha evidenciado disminución significativa del conteo plaquetario después de administrar Clopidogrel en una sola dosis ${ }^{7}$, pero siempre manteniéndose dentro de valores considerados como normales. Otros investigadores sin embargo han reportado modificaciones sobre los leucocitos y eritrocitos, pero no sobre las plaquetas ${ }^{6}$.

Dado que las plaquetas tienen una alta concentración de FCs que pueden utilizarse para acelerar los procesos de regeneración tisular cuando sea requerido por el paciente, el estudio de la acción de los antiagregantes plaquetarios y su relación con el recuento plaquetario, permitirá inferir si estos medicamentos modifican la cantidad de plaquetas de manera importante, que dificulten el empleo del PRP en pacientes que reciben estos medicamentos. De allí que el objetivo de esta investigación fue evaluar el recuento plaquetario en sujetos sanos tratados con antiagregantes plaquetarios durante 7 días.

\section{MATERIALES Y MÉTODOS}

\section{Tipo y Diseño de investigación}

Esta es una investigación descriptiva, de campo, no experimental y longitudinal ${ }^{14}$.

\section{Población y muestra}

Estuvo conformada por sujetos adultos, de sexo femenino y masculino, atendidos en la consulta de medicina general del Instituto de Investigaciones Clínicas (IIC) de La Universidad del Zulia, Venezuela, durante el periodo febrero del 2014 a febrero del 2015. La muestra fue de tipo no probabilístico, de conveniencia o intencional ${ }^{15}$.

\section{Criterios de selección}

Se incluyeron sujetos entre 18 y 50 años de edad, de ambos sexos, que desearon participar en este estudio, sin enfermedad clínica de base conocida, aparentemente sana (previa valoración clínica general) y con resultados normales en el estudio de agregación plaquetaria antes del tratamiento con antiagregantes plaquetarios y finalmente que acataron de manera estricta el tratamiento con los antiagregantes indicados. Fueron excluidos aquellos que habían ingerido previamente antiagregantes plaquetarios o bebidas alcohólicas 11 días antes del estudio, o que estuvieran recibiendo cualquier tipo de tratamiento.

\section{Procedimiento}

Se incluyeron 20 sujetos, 13 femeninos y 7 masculinos, y se distribuyeron en dos grupos, de la siguiente manera: Grupo A: 20 sujetos sin tratamiento previo con drogas antiagregantes plaquetarias; estos mismos conformaron el Grupo B, pero que a su vez fueron divididos en dos subgrupos: B1: 10 sujetos tratados con Aspirina a una dosis de $100 \mathrm{mg}$ diarios por 7 días y $\underline{\mathrm{B} 2}$ : 10 individuos quienes recibieron Clopidogrel a una dosis de $75 \mathrm{mg}$ diarios por 7 días.

Los sujetos debían encontrarse en ayunas, se les extrajo por punción venosa $16 \mathrm{ml}$ de sangre, empleando catéter pericraneal $\mathrm{N}^{\circ} 21$ siguiendo la técnica de la doble jeringa para evitar la activación de las plaquetas ${ }^{16}$. Se empleó una jeringa con la cual se extrajo $2,5 \mathrm{ml}$ de sangre que se trasegó a un tubo de vidrio que contenía EDTA y que se utilizó para hacer el conteo inicial de plaquetas, empleando un contador automático de células sanguíneas marca Beckman Coulter AC-T. La segunda jeringa contenía $13,5 \mathrm{ml}$ de sangre venosa que se distribuyeron en dos tubos plásticos que contenían de citrato de sodio al 3,8\% (9/1): en uno se vertió $9 \mathrm{ml}$ y en el otro 4,5 ml; en ambos se realizó estudio de agregación plaquetaria, empleando un agregómetro Chrono-log (Corp. Haverton, PA, USA). En cada una de estas muestras también se realizó el conteo plaquetario.

Los tubos con citrato de sodio, fueron sometidos a centrifugación en centrifuga clínica. El que contenía 9 $\mathrm{ml}$ de sangre, se dejó reposar 20 minutos, y luego fue centrifugado a una velocidad de $1.400 \mathrm{rpm}$ por 7 minutos, siguiendo la técnica de Anitua ${ }^{17}$. El tubo con $4,5 \mathrm{ml}$ de sangre se sometió a $800 \mathrm{rpm}$ durante 10 minutos a temperatura ambiente. Lo que quedó de cada tubo, se centrifugó de nuevo a $4.500 \mathrm{rpm}$ durante 20 minutos, en centrífuga refrigerada (Sorvall), para obtener Plasma Pobre en Plaquetas (PPP). De igual manera, en estas muestras también se realizó conteo plaquetario. 
Se realizó un ajuste de la concentración plaquetaria a 250 x $10^{9} / 1$ con el PPP, antes de llevarse a cabo la agregación respectiva y el recuento de plaquetas se ajustó antes del tratamiento con los antiagregantes plaquetarios ya mencionados. Para la realización de la prueba de agregación se empleó el método turbidimétrico de Born ${ }^{18}$ y el agregómetro Chrono-log (Corp. Haverton, PA, USA). De manera que a $0,45 \mathrm{ml}$ de las plaquetas ajustadas se les adicionó los siguientes inductores: ADP (Adenosina 5 Difosfato): 5 × 10-6 M (concentración final: $2,14 \mu \mathrm{g} / \mathrm{mlL}$ ), colágeno: $1,2 \times 10^{-2} \mathrm{M}$ (concentración final: $3 \mu \mathrm{g} / \mathrm{ml}$ ) y epinefrina: 0,6 x $10^{-4} \mathrm{M}$ (concentración final: $11 \mu \mathrm{g} / \mathrm{ml}$ ). El resultado obtenido se expresó en porcentaje.

Posteriormente, a cada sujeto se le instruyó consumir la dosis correspondiente al antiagregante respectivo: $100 \mathrm{mg}$ de AAS o $75 \mathrm{mg}$ de Clopidogrel en ayunas, por 7 días, al cabo de los cuales se realizó nuevamente la extracción sanguínea para el conteo plaquetario, siguiendo el procedimiento ya descrito.

\section{Valores Referenciales}

Se tomaron en cuenta los valores de referencia para el recuento plaquetario entre 150,0 a 450,0 × $10^{9} \mathrm{l}$ en sangre periférica; y un conteo de plaquetas en el PRP de 2 a 5 veces superior al número de plaquetas que se obtuvo en sangre periférica ${ }^{5}$. Mientras que para la agregación plaquetaria en cada agonista utilizado se consideró como valores normales para ADP de $68 \%-88 \%$, Colágeno entre $70-94 \%$ y Epinefrina entre 78 a $88 \%{ }^{19}$.

\section{Técnicas de procesamiento y análisis de datos}

Los datos se muestran en tabla y gráficos. Se aplicó la prueba t student (datos no pareados) para la comparación de medias y para medir el efecto de cada uno de los tratamientos (Aspirina vs Clopidogrel). Así mismo, se empleó la estadística descriptiva para determinar el recuento plaquetario antes y después de los tratamientos. Se trabajó con intervalos de confianza del $95 \%$, por ello se consideró significativo los valores de $\mathrm{p}<0,05$. Para todos los análisis se utilizó el software estadístico SPSS18 20,21.

\section{Declaración de aspectos éticos}

A todos los sujetos objeto de estudio se les informó acerca de los objetivos, detalles procedimentales y alcance de esta investigación, y se les hizo firmar un consentimiento informado por escrito, que conto con la aprobación de la Comisión de Ética de la Institución de investigación y acatando los principios de la declaración de Helsinki de 1975 (actualizada en el 2013) ${ }^{22}$, y a las recomendaciones elaboradas por el Consejo de Organizaciones Internacionales de Ciencias Médicas (CIOMS) en el $2002^{23}$.

\section{RESULTADOS}

Se observa que el contaje de plaquetas fue similar tanto en sangre periférica como en el PRP antes y después del tratamiento recibido (Tabla 1). Se produjo un incremento significativo en el PRP con respecto a la sangre periférica, tanto en condiciones basales como después del tratamiento con este antiagregante (basal $\mathrm{p}$ $<0,05$, postratamiento $<0,0001$ ), pero todos los valores se encuentran dentro del rango referencial.

Con respecto a los resultados obtenidos y mostrados en la Tabla 2 en los sujetos tratados con Clopidogrel, se observó una disminución significativa $(p<0,05)$ en sangre periférica después de recibir este antiagregante. Similar al comportamiento del grupo tratado con AAS se observó al comparar el recuento plaquetario de sangre periférica versus PRP (antes y después del tratamiento), pues hubo un incremento significativo tanto en condiciones basales, como postratamiento (Basal: $\mathrm{p}<0,05$, postratamiento: $\mathrm{p}<0,0001)$.

Cuando se comparó el contaje plaquetario en los sujetos tratados con AAS y Clopidogrel tanto en sangre periférica como en PRP, se observó que en condiciones basales mostraron valores similares (Tabla 3), no así posterior al tratamiento, donde se observó una disminución significativa $(<0,005)$ del recuento plaquetario en sangre periférica en aquellos individuos tratados con Clopidogrel (Tabla 4). 
Tabla 1. Valores promedios, desviación estándar y rango del recuento plaquetario en sangre periférica y PRP de sujetos sanos antes y después del tratamiento con AAS. Instituto de Investigaciones Clínicas Dr. Américo Negrette.

LUZ. 2015.

\begin{tabular}{|c|c|c|c|}
\hline \multirow{2}{*}{$\begin{array}{c}\text { Muestra } \\
\text { Sanguínea }\end{array}$} & BASAL & Plaquetas $\left(\mathrm{x} 10^{9} \mathrm{l}\right)$ & \multirow{2}{*}{$\mathrm{P}$} \\
\cline { 2 - 4 } & $258,60 \pm 54,46$ & $254 \pm 41,86$ & NS \\
\hline Sangre Periférica & $486,50 \pm 129,54$ & $449,20 \pm 85,51$ & NS \\
\hline Plasma Rico en Plaquetas & $<0,05$ & $<0,0001$ & \\
\hline P & $<$
\end{tabular}

AAS: Aspirina. p: Significancia. NS: No significativo

Tabla 2. Valores promedios, desviación estándar y rango del recuento plaquetario en sangre periférica y PRP de sujetos sanos antes y después del tratamiento con Clopidogrel. Instituto de Investigaciones Clínicas Dr. Américo Negrette. LUZ. 2015.

\begin{tabular}{|c|c|c|c|}
\hline \multirow{2}{*}{$\begin{array}{c}\text { Muestra } \\
\text { Sanguínea }\end{array}$} & Basal & Claquetas $\left(\mathrm{x} 10^{9} \mathrm{l}\right)$ & \multirow{2}{*}{$\mathrm{P}$} \\
\cline { 2 - 3 } & $285,40 \pm 70,92$ & $196,50 \pm 37,90$ & $<0,05$ \\
\hline Sangre Periférica & $565,20 \pm 150,41$ & $592,90 \pm 203,46$ & NS \\
\hline Plasma Rico en Plaquetas & $<0,05$ & $<0,0001$ & \\
\hline P & $<$ & & \\
\hline
\end{tabular}

p: Significancia. NS: No significativo

Tabla 3. Valores promedios, desviación estándar y rango del recuento plaquetario en sangre periférica y PRP, de sujetos sanos antes del tratamiento con AAS versus Clopidogrel. Instituto de Investigaciones Clínicas Dr. Américo Negrette. LUZ. 2015.

\begin{tabular}{|c|c|c|c|}
\hline \multirow{2}{*}{ Muestra Sanguínea } & \multicolumn{2}{|c|}{ Plaquetas (x 10 1 l) Basal } & \multirow{2}{*}{ P } \\
\cline { 2 - 3 } & Con AAS & Con Clopidogrel & \multirow{2}{*}{ NS } \\
\hline Sangre Periférica & $258,60 \pm 54,46$ & $285,40 \pm 70,92$ & NS \\
\hline Plasma Rico en Plaquetas & $486,50 \pm 129,54$ & $565,20 \pm 150,41$ & \\
\hline P & $<0,0001$ & $<0,0001$ & \\
\hline
\end{tabular}

AAS: Aspirina. p: Significancia. NS: No significativo 
Tabla 4. Valores promedios, desviación estándar y rango del recuento plaquetario en sangre periférica y PRP, de sujetos sanos después del tratamiento con AAS versus Clopidogrel. Instituto de Investigaciones Clínicas Dr. Américo Negrette. LUZ. 2015.

\begin{tabular}{|c|c|c|c|}
\hline \multirow{2}{*}{$\begin{array}{c}\text { Muestra } \\
\text { Sanguínea }\end{array}$} & \multicolumn{2}{|c|}{ Plaquetas $(\mathrm{x} \mathrm{10}$ l) con Antiagregantes } & P \\
\hline Sangre Periférica & $254 \pm 41,86$ & $196,50 \pm 37,90$ & $<0,005$ \\
\hline Plasma Rico en Plaquetas & $449,20 \pm 85,51$ & $592,90 \pm 203,46$ & NS \\
\hline P & $<0,0001$ & 0,0001 & \\
\hline
\end{tabular}

AAS: Aspirina. p: Significancia. NS: No significativo

\section{DISCUSIÓN}

En la presente investigación se encontró que, antes del tratamiento con AAS y Clopidogrel, el conteo plaquetario en sangre periférica fue $258,60 \pm 54,46 \times 10^{9}$ /litro y en el PRP 486,50 $\pm 129,54 \times 10^{9} / 1$ para el primero; mientras que para el segundo fue 285,40 $\pm 70,92 \times 10^{9} \mathrm{l}$ en sangre periférica y 565,20 $\pm 150,41 \times 10^{9} \mathrm{l}$ en el PRP, observándose diferencia estadísticamente significativa $(\mathrm{p}<0,05)$ en ambos casos. Esto representó un incremento plaquetario de 1,9 veces para la AAS y 1,98 veces para el Clopidogrel. Este comportamiento es el esperado luego de someter la muestra sanguínea a centrifugación utilizando el protocolo de Anitua, de esta manera se obtiene un verdadero PRP con una concentración efectiva de plaquetas, aportando una gran concentración de $\mathrm{FCs}^{17}$.

Los valores en sangre periférica son similares a los obtenidos por otros autores, entre los que se destaca Weibrich et $\mathrm{a}^{24} \mathrm{y}$ Agren et $\mathrm{al}^{25}$; el primero determinó la concentración de factores de crecimiento en el PRP y obtuvieron un conteo plaquetario de $260 \pm 57,5 \times 10^{3} \mathrm{x}$ $\mathrm{mm}^{3}$, mientras que el segundo cuantificó los factores de crecimiento en el PRF (Plasma Rico en Fibrina: derivado del PRP) y las cifras plaquetarias fueron de $253 \pm 47 \mathrm{x}$ $10^{3} \mathrm{x} \mathrm{mm}^{3}$.

Al analizar el comportamiento del contaje de plaquetas en sangre periférica antes y 7 días después del tratamiento con los antiagregantes plaquetarios utilizados, se observó que para la AAS los valores fueron similares $\left(258,60 \pm 54,46 \times 10^{9}\right.$ /litro versus $254 \pm 41,86 \times 10^{9}$ / litro, sin diferencias significativas); mientras que para el Clopidogrel hubo un descenso significativo $(\mathrm{p}<0,05)$ $\left(285,40 \pm 70,92 \times 10^{9} / \mathrm{l}\right.$ versus $\left.196,50 \pm 37,90 \times 10^{9} / 1\right)$.
Dichos resultados coinciden con los reportados por González et $\mathrm{al}^{7}$ quienes estudiaron sujetos sanos tratados con Aspirina (100 mg) y Clopidogrel (75 mg) pero en dosis única. No así con los resultados presentados por Fontana et $\mathrm{al}^{26}$, quienes midieron los efectos biológicos de la aspirina y el Clopidogrel en sujetos sanos, no encontrando diferencias significativas en sangre periférica para el recuento plaquetario luego de la administración por una semana de ambos fármacos.

Cuando se enfrentaron los resultados observados para el recuento plaquetario en sangre periférica luego del tratamiento con AAS vs Clopidogrel durante 7 días (254 $\pm 41,86 \times 10^{9} 1$ vs $196,5 \pm 37,9 \times 10^{9} 1$, respectivamente) se observó diferencias estadísticamente significativas $(\mathrm{p}<$ 0,05), pero no para el PRP, discrepando con lo reportado por González et al. ${ }^{27}$ para el PRP cuando se utilizaron los tratamientos antes descritos.

Estos hallazgos parecen reafirmar que en nuestra investigación se experimentó una disminución significativa en el recuento plaquetario en la sangre periférica, luego del tratamiento con Clopidogrel durante 7 días, pero esta disminución no se sitúo por debajo del rango referencial ( $\left.<150,0 \times 10^{9} \mathrm{l}\right)$, lo que permite inferir que el Clopidogrel pareció afectar el número de las plaquetas primordialmente en sangre periférica pero no en el PRP. Esto podría deberse al recambio plaquetario que se genera en condiciones normales después de la administración de estas drogas, que es de aproximadamente 7 días $^{8}$.

El comportamiento observado en cuanto al conteo plaquetario en sangre periférica en los sujetos tratados con AAS o Clopidogrel, llama la atención sobre el control y vigilancia que se debería llevar a cabo en 
sujetos con patologías que ameritan de tratamientos con antiagregantes plaquetarios, sobre todo si estos son prolongados. Por ello se requiere de un estudio similar, con un mayor número de individuos.

Existe el falso entendimiento de que los fármacos antiagregantes plaquetarios pueden afectar significativamente tanto el número de plaquetas como su contenido en factores de crecimiento, lo que a su vez alteraría la capacidad regenerativa de este derivado plaquetario utilizado en tratamientos de diversas patologías médicas. Estos resultados indican que las cifras de plaquetas obtenidas en el PRP luego de 7 días de tratamiento con AAS o Clopidogrel, no sufrieron cambios significativos, por lo que puede considerarse el empleo de ese PRP en sujetos tratados con antiagregantes, para acelerar la regeneración tisular en caso de ameritarlo.

En resumen, el presente trabajo permitió evaluar el recuento plaquetario en SP y PRP de sujetos sanos antes y 7 días después de la administración de antiagregantes plaquetarios, hallándose que el AAS no afectó el recuento plaquetario de manera significativa, pero si para el Clopidogrel el cual mostró una disminución significativa en SP 7 días después de su administración encontrándose, sin embargo, dentro del rango referencial. Se recomienda la realización de nuevos estudios con un mayor número de muestras provenientes de sujetos sometidos a tratamiento con diversos antiagregantes plaquetarios.

\section{DECLARACIÓN SOBRE CONFLICTO DE INTERESES}

Los autores de este manuscrito declaran no tener ningún tipo de conflicto de intereses.

\section{REFERENCIAS BIBLIOGRÁFICAS}

1. Bizzozero J. On a new blood particle and its role in thrombosis and blood coagulation. Virchows Arch B Cell Pathol Incl Mol Pathol. 1982; 90: 261.

2. Im MJ, Kim YS, Edwards RJ, Hoopes JE, Fenselau A. The effect of bovine basic fibroblast growth factor on skin flap survival in rats. Ann Plast Surg 1992; 28 (3): 242-245.

3. Adler SC, Kent KJ. Enhancing wound healing with growth factors. Facial Plast Surg Clin North Am. 2002; 10 (2): 129-46.

4. González-Lagunas J. Plasma rico en plaquetas Rev Esp Cir Oral Maxilofac 2006; 28 (2): 89-99.
5. Sánchez AR, Sheridan PJ, Kupp LI. Is platelet-rich plasma the perfect enhancement factor? A current review. Int J Oral Maxillofac Implants 2003; 18(1): 93-103

6. Hayasaka M, Takahashi Y, Nishida Y, Yoshida Y, Hidaka S, Asai S. Comparative effect of clopidogrel plus aspirin and aspirin monotherapy on hematological parameters using propensity score matching. Vasc Health Risk Manag 2013; 9: 65-70.

7. González M, Arteaga-Vizcaíno M, Ruiz A, Daniela E, González B, Galué R, et al. Acción de los antiagregantes plaquetarios sobre el recuento de plaquetas en el plasma rico en plaquetas. ACHO. 2013; 2 (2): 15-22.

8. Patrono C, Bachmann F, Baigent C, Bode C, De Caterina R, Charbonnier B, et al. Grupo de trabajo sobre el uso de agentes antiplaquetarios en pacientes con enfermedad cardiovascular aterosclerótica de la Sociedad Europea de Cardiología. Documento de Consenso de Expertos sobre el uso de agentes antiplaquetarios. Rev Esp Cardiol. 2004; 57(10): 963-80.

9. Ruland S. Safety of Clopidogrel and Aspirin for stroke Prevention: Implications of the CHARISMA Trial. Drug Saf. 2008; 31(6):449-458.

10. Tanasescu S, Lévesque H, Thuillez C. Farmacología de la Aspirina. Rev Med Int 2000; 21; 1: 18s-26s.

11. Hilman R, Kenneth A, Henry M. Hematología en la práctica clínica. $4^{\mathrm{a}}$ edic. Editorial Manual Moderno; 2005.

12. CAPRIE Steering Committee. A randomized, blinded, trial of clopidogrel versus aspirin in patients at risk of ischaemic events (CAPRIE). Lancet. 1996; 348(9038):1329-1339.

13. Sharis PJ, Loscalzo J. Thienopyridines: ticlopidine and clopidogrel. In: Sasahara A, Loscalzo J, editors. New Therapeutic Agents in Thrombosis and Thrombolysis, 2nd ed. New York, NY: Informa Healthcare; 2003.

14. Hernández Sampieri R, Fernández Collado C, Baptista LP. Metodología de la Investigación. Cuarta Edición México D.F; Editorial Mc Graw Hill. 2006.

15. Arias Fidias G. El Proyecto de Investigación, Introducción a la Metodología Científica Quinta Edición. CaracasVenezuela; Editorial Episteme; 2006.

16. Symansky, MR., Fox, HA. Umbilical vessel catheterization: Indications, management, and evaluation of the thecnique. J Pediatr. 1972; 80(5): 820-826.

17. Anitua E. La utilización de los factores de crecimiento plasmáticos en cirugía oral, maxilofacial y periodoncia (PRGF). RCO 2001; 6: 305-15.

18. Born GVR, Cross MJ. The aggregation of the blood platelets. J Phisiol 1963; 168(1): 178-183.

19. Bick RL. Platelet function defects. In: Bick RL, editor. Disorders of thrombosis and hemostasis: clinical and laboratory practice. USA: Lippincott Williams \& Wilkins; 2002. p. 59-90. 
20. Magdalena F. Análisis Estadístico. Primera Edición Madrid. Editorial Osborne MacGrawHill. 2001.

21. Pardo A, San Martin R. Análisis de Datos en Ciencias Sociales y de Salud II. $2^{\text {da }}$ edición. Madrid; Editorial Síntesis. Madrid. 2010.

22. Declaración de Helsinki de la AMM - Principios éticos para las investigaciones médicas en seres humanos (Internet). (Citado 18 Jun 2014). Disponible en: http:// www.wma.net/es/30publications/10policies/b3/.

23. CIOMS working group on the revision on 2002 CIOMS Ethical guidelines for Biomedical research (Internet). (Citado 29 Oct 2015). Disponible en: http://www. cioms.ch/index.php/2012-06-10-08-47-53/ethics/ciomsguidelines-working-group.

24. Weibrich W, Kleis W, Hafner G. Growth factor levels in the platelet-rich plasma produced by 2 different methods: Curasan-type PRP kit versus PCCS PRP system. Int J Or Maxill Im 2002; 17(2): 184-190.

25. Agren MS, Rasmussen K, Pakkenberg B, Jorgensen B. Growth factor and proteinase profile Vivostat ${ }^{\circledR}$ plateletrich fibrin linked to tissue repair. Vox Sang 2014; 107(1): 37-43.

26. Fontana P, Nolli S, Reber G De Moerlosse P. Biological effects of aspirin and clopidogrel in a randomized crossover study in 96 healthy volunteers. J Throm Haemost 2006; 4(4): 813-819.

27. González M, Arteaga-Vizcaíno M, Ruiz A, Briceño O, Quintero M, Atencio R, et al. Niveles del factor de crecimiento derivado de plaquetas en el plasma rico en plaquetas antes y después de antiagregantes plaquetarios Avan Biomed 2013; (2): 19-23.

Para citar este artículo: González-Rincón M, Arteaga-Vizcaíno M, Ruíz A, Briceño O, Quintero M, Quintero J, et al. Conteo de plaquetas en sujetos sanos tratados con antiagregantes plaquetario. Duazary. 2016 julio; 13 (2): 87 - 94 